\title{
CARACTERIZAÇÃO ELETROQUÍMICA DE FILMES ZrN E ZrSiN DEPOSITADOS SOBRE O AÇO INOXIDÁVEL 316L APÓS UM LONGO PERÍODO DE IMERSÃO EM SOLUÇÃO SALINA
}

\section{ELECTROCHEMICAL CHARACTERIZATION OF ZrN AND ZrSiN COATINGS ON 316L STAINLESS STEEL AFTER A LONG TIME OF IMMERSION IN SALINE SOLUTION}

\author{
F.L. Menezes ${ }^{1}$ *, E.K. Tentardin ${ }^{2}$, M.M.R. Castro $^{1}$
}

1 Universidade Federal de Minas Gerais, Belo Horizonte, MG, 31270-901, Brasil.

2 Universidade Federal de Sergipe, São Cristóvão, SE 49100-000, Brasil.

*Corresponding author: Universidade Federal de Minas Gerais, Belo Horizonte, Minas Gerais, 31270-901, Brasil. Phone: +55982394400.

E-mail:Im_fernanda@yahoo.com.br(F.L.Menezes).

\begin{tabular}{l} 
A R T I C L E I N F O \\
\hline Article history: \\
Received 2019-04-11 \\
Accepted 2019-06-27 \\
Available online 2019-06-30 \\
p a l a vra s-chave \\
Corrosão \\
Aço inoxidável \\
Nanodureza \\
ZrN \\
ZrSiN \\
ke ywords \\
Corrosion \\
Stainless steel \\
Nanohardness \\
ZrN \\
ZrSiN \\
\end{tabular}

\begin{abstract}
A B S T R A C T
The use of coatings to improving the material properties is an alternative for the development of new materials. ZrN and ZrSiN films are known for their good mechanical properties. However, there are just few researches that investigate their corrosion resistance. In this way, this work studied the corrosion resistance of the $\mathrm{ZrN}$ and $\mathrm{ZrSiN}$ films deposited on 316L stainless steel, immersed in a $3.0 \% \mathrm{~m} / \mathrm{v} \mathrm{NaCl}$ solution for a $1000 \mathrm{~h}$ period. Corrosion tests were carried out using Electrochemical Impedance Spectrometry. The nanohardness technique was used to evaluate the hardness. The films' morphology was analyzed by Scanning Electron Microscopy, before and after $1000 \mathrm{~h}$ of immersion. The results showed that $\mathrm{ZrN}$ and ZrSiN films improved the corrosive properties of $316 \mathrm{~L}$ stainless steel. The 2.5 at. $\%$ Si addition enhanced the corrosion resistance but not the hardness of $\mathrm{ZrN}$ coat.
\end{abstract}

R E S U M O

A utilização de revestimentos é uma forma de melhorar as propriedades de um material. Os filmes $\mathrm{ZrN}$ e ZrSiN são conhecidos pelas suas boas propriedades mecânicas, porém existem poucas pesquisas que investiguem a resistência à corrosão dos mesmos. Dessa forma, por meio da técnica de Espectrometria de Impedância Eletroquímica, estudou-se a resistência à corrosão dos filmes de ZrN e ZrSiN depositados no aço inoxidável 316L imersos em uma solução de $\mathrm{NaCl} 3,0 \% \mathrm{~m} / \mathrm{v}$, por um período de $1000 \mathrm{~h}$. A técnica de nanodureza foi aplicada para avaliar a dureza. A morfologia dos filmes, antes e após o teste de imersão, foi analisada por Microscopia Eletrônica de Varredura. Observou-se que os filmes de $\mathrm{ZrN}$ e ZrSiN melhoraram as propriedades corrosivas do aço 316L. A adição de 2,5\% at. de Si ao filme ZrN melhorou a resistência à corrosão, mas não aumentou a dureza do recobrimento. 


\section{INTRODUÇ̃̃̃O}

O aumento da vida útil de equipamentos e materiais tem grande importância tanto do ponto de vista financeiro quanto ambiental. Um dos principais motivos pela diminuição da durabilidade dos materiais é a corrosão. Nesse sentido, os revestimentos são importantes alternativas como meio de proteção, na medida que isolam o substrato do meio agressivo. Os revestimentos cerâmicos, como os carbonetos e nitretos dos elementos de transisão do grupo IV, são vistos como boa opção de filmes, devido sua alta dureza, alta temperatura de fusão e grande resistência à oxidação (Harrison e Lee, 2016).

Alat et al (2015) estudaram os revestimentos TiAlN e TiN para aplicação em reatores de combustíveis nucleares, em substituição as ligas de zircônio. Cubillos et al. (2014) estudaram a influência da temperatura do substrato na estrutura, morfologia e resistência à corrosão para processo de deposição do filme $\mathrm{ZrOxNy} / \mathrm{ZrO} 2$ por $\mathrm{RF}$ sputtering. Os autores verificaram que aumento da temperatura do aço inoxidável 304 como substrato aumenta a rugosidade do revestimento e reduz o tamanho de partícula do mesmo. Isto diminui a permeabilidade da solução corrosiva através dos contornos de grão, o que reflete na maior resistência à corrosão do revestimento a $350^{\circ} \mathrm{C}$. Milani (2009) estudou a nitretação da zircônia, formando uma camada de nitreto de zircônio, e concluiu que a dureza e a condução de eletricidade foram melhoradas.

De acordo com sua aplicação, um revestimento deve ter uma série de propriedades como boa aderência ao substrato, estabilidade térmica em altas temperaturas, resistência ao desgaste e à corrosão. Essas características estão relacionadas com os parâmetros de deposição, que variam conforme a técnica empregada. Nesse trabalho, os filmes finos $\mathrm{ZrN}$ e $\mathrm{ZrSiN}$ foram depositados em aço inoxidável 316L pela técnica de magnetron sputtering. Essa é uma técnica do tipo PVD (Physical Vapour Deposition), que possibilita a produção de filmes com estequiométria e composição controlável, uniforme, com alta taxa de deposição e em escala industrial (Safi, 2000).

Revestimentos à base de $\mathrm{ZrN}$ são aplicados em condições que requerem boa resistência ao desgaste, como ferramentas de corte de metal, e alta estabilidade térmica. Porém, os filmes de $\mathrm{ZrN}$ não possuem boa resitência à oxidação (Yalamanchili et al., 2016). ZrN possue uma estrutura cúbica de face centrada (CFC), cor predominantemente dourada e microsestrutura colunar (Harrison e Lee, 2016). A estrutura colunar favorece a formação de poros e trincas, o que permite o contato do meio eletroquímico mais facilmente com o subtrato, limitando a aplicação deste tipo de recobrimento. A adição de um terceiro elemento, como o silício, constitui uma alternativa para melhorar as características estruturais do filme de $\mathrm{ZrN}$ (Freitas et al., 2015).

Neto et al. (2018) estudaram a microestrutura do filme $\mathrm{ZrSiN}$ com concentrações de silício variando entre $0-15 \%$ at. (porcentagem atômica). Também estudaram a resistência a oxidação do filme nas temperaturas de $773 \mathrm{~K}, 873 \mathrm{~K}, 973 \mathrm{~K} \mathrm{e}$ 1073 K. Em seu trabalho, Neto et al. (2018) confirmaram que a adição de silício resulta em um filme com estrutura binária, parte cristalina $(\mathrm{ZrN})$ e parte amorfa $\left(\mathrm{Si}_{3} \mathrm{~N}_{4}\right)$. Essa diferença na microestrutura dos filmes de $\mathrm{ZrN}$ e $\mathrm{ZrSiN}$ resulta em um filme com maior resistência a oxidação e maior dureza. Cui et al. (2013) avaliaram a microestrutura e resistência à corrosão do revestimento $\mathrm{ZrSiN}$, depositado pela técnica de magnetron sputtering, em liga de titânio, em meio de albumina a $37^{\circ} \mathrm{C}$. Os autores comprovaram, por meio da espectroscopia de impedância eletroquímica, que existe uma concentração ótima para o silício, no caso $8,32 \%$ at. de $\mathrm{Si}$, acima da qual a resistência à corrosão diminui. Mae, et al. (2001) avaliaram o efeito da adição de Si no filme de $\mathrm{ZrN}$, depositado pela técnica de magnetron sputtering em substratos de silício e alumínio. A concentração de silício variou em 0 a $12,3 \%$ at. Os autores concluíram que adição de silício aumenta a dureza do filme até um valor limite, a partir do qual há uma diminuição da mesma.

Devido a grande aplicação dos aços inoxidáveis, esse artigo avalia qualitativamente a resistência à corrosão eletroquímica dos filmes $\mathrm{ZrN}$ e $\mathrm{ZrSiN}$ depositados no aço inoxidável 316L em meio de $\mathrm{NaCl} 3,0 \% \mathrm{~m} / \mathrm{v}$, à temperatura ambiente. Para tal, utilizou-se a técnica de Espectroscopia de Impedância Eletroquímica (EIE) durante um tempo de imersão de $1000 \mathrm{~h}$. A investigação das propriedades de corrosão em um longo tempo de imersão não é comum, mas tem sido cada vez mais aplicada por fornecer informações utéis relacionadas à proteção contra a corrosão efetiva dos revestimentos. Além disso, avaliou-se a dureza, utilizando a técnica de nanodureza, e a superfície morfológica do filme, por meio da análise de Microscopia Eletrônica de Varredura (MEV). A avaliação qualitativa da resistência à corrosão eletroquímica de filmes $\mathrm{ZrN}$ e $\mathrm{ZrSiN}$ depositados no aço inoxidável $316 \mathrm{~L} \mathrm{em}$ meio de $\mathrm{NaCl}$ $3,0 \% \mathrm{~m} / \mathrm{v}$, a temperatura ambiente, é uma contribuição importante para os estudos constantes de inibição de corrosão.

\section{MATERIAIS E MÉTODOS}

O aço inoxidável 316L (fornecido pela Aperam South American) foi utilizado como substrato para os revestimentos. A composição química nominal desse aço é mostrada na tabela 1. As amostras do aço inoxidável 316L foram cortadas em tamanhos de aproximadamente $50 \mathrm{~mm} \times 50 \mathrm{~mm} \times 2 \mathrm{~mm}$. Em seguida foram lixadas em lixadeiras mecânicas utilizando papeis de $\mathrm{SiC}$ de número 100, 220, 320, 400 e 600 mesh, nessa ordem. Após serem lixadas, as amostras foram lavadas com álcool e acetona, para remoção de gordura, e secas com secador.

Após a preparação da superfície, as amostras foram revestidas com os filmes de $\mathrm{ZrN}$ e $\mathrm{ZrSiN}$ por meio da técnica magnetron sputtering utilizando o equipamento Orion 5-HV Sputtering Systems, da empresa AJA International. Os filmes foram depositados sobre o substrato de aço inoxidável 316L com pressão de trabalho a 3 mtorr, relação $\mathrm{Ar} / \mathrm{N}_{2}$ de 19/2, potência de $120 \mathrm{~W}$ no alvo de zircônio e $50 \mathrm{~W}$ no alvo de silício (para uma concentração atômica de 2,5 \% at. de Si). Não houve adição de temperatura externa no interior da câmara. Ambos os filmes de $\mathrm{ZrN}$ e $\mathrm{ZrSiN}$ possuem espessura de aproximadamente $200 \mathrm{~nm}$.

Os testes de espectrocopia de impedância eletroquímica (EIE) foram conduzidos em um potenciostato da marca Gamry Instruments, serie G300. A célula eletroquímica foi montada com três eletrodos, sendo um eletrodo de trabalho (amostras dos aços com e sem revestimento), um eletrodo de referência $\mathrm{Ag} / \mathrm{AgCl}(4 \mathrm{M} \mathrm{KCl},+0,200 \mathrm{~V}$ vs. SHE) e um contra eletrodo de grafite. Em todas as análises, a área exposta das amostras em contato com a solução eletrolítica de $\mathrm{NaCl} 3,0 \% \mathrm{~m} / \mathrm{v}$ foi de 2,85 $\mathrm{cm}^{2}$. Os testes de EIE tiveram amplitude de perturbação de $10 \mathrm{mV}$ em torno do OCP e faixa de frequência de $10 \mathrm{mHz}$ a 100 $\mathrm{kHz}$. A resistência de cada filme foi monitorada e medida após $1,2,24,168,336,504,840$ e $1000 \mathrm{~h}$. Antes de cada medição de 
EIE, o potencial de circuito aberto (OCP) foi medido por $1800 \mathrm{~h}$ ou até o potencial se tornar estável (uma variação máxima de 5 $\mathrm{mV}$ em $5 \mathrm{~min}$ ). Os diagramas de Nyquist foram plotados no software Origin ${ }^{\circledR} 8.0$.
A medida de dureza foi realizada pelo equipamento Hysitron Tribo Indenter 900 com indentação máxima de $25 \mathrm{~nm}$. Antes e após os testes de EIE, analisou-se a superfície da amostra por miscroscopia eletrônica de varredura utilizando o equipamento FEG - FEI Quanta 200.

Tabela 1 - Composição química nominal do aço AISI 316L em porcentagem.

\begin{tabular}{ccccccccc} 
Amostra & $\mathrm{C}$ & $\mathrm{Mn}$ & $\mathrm{Si}$ & $\mathrm{Cr}$ & $\mathrm{Ni}$ & $\mathrm{Cu}$ & $\mathrm{Mo}$ & $\mathrm{N}_{2}$ \\
\hline 316L & 0,0194 & 1,3931 & 0,4658 & 16,593 & 10,005 & 0,1368 & 2,0343 & 0,0390
\end{tabular}

Fonte: APERAM

\section{RESULTADOS E DISCUSSÃO}

Na figura 1 é apresentada a evolução do potencial de circuito aberto (OCP) para o substrato com e sem revestimento, após $1000 \mathrm{~h}$ de imersão. O aço inoxidável 316L sem revestimento é o que possui menor OCP médio e foi a amostra que apresentou uma menor taxa de cresimento do potencial com o tempo. A amostra com revestimento de $\mathrm{ZrN}$ teve um aumento do potencial com o tempo, porém com uma taxa de aumento do potencial menor se comparado com o filme de ZrSiN. Já o sistema substrato - $\mathrm{ZrSiN}$ possui maior valor de OCP médio, uma maior taxa de crescimento do potencial, o que indica uma tendência a maior resistência à corrosão ou uma maior nobreza do sistema em termos termodinâmicos.

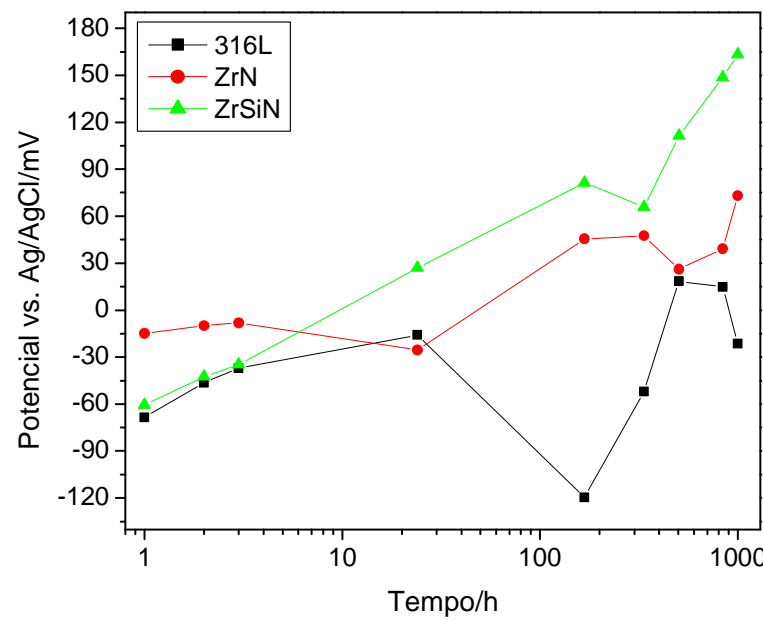

Figura 1 - Potencial de circuito aberto das amostras investigadas em solução de $\mathrm{NaCl} 3,0 \% \mathrm{~m} / \mathrm{v}$.

Os diagramas de Nyquist apresentados nas figuras 2, $3 \mathrm{e}$ 4 mostram um aumento no diâmetro da curva com o passar do tempo. Segundo Lin e Duh (2009) existe uma tendência de que, para o diagrama de Nyquist, quanto maior o diâmetro da curva, maior a resistência à corrosão do material. Dessa forma, podese afirmar que a resistência à corrosão aumenta com o tempo para o substrato com e sem revestimento. Para o aço inoxidável 316L sem revetimento, o aumento da resistência à corrosão se deve à formação da camada de passivação. Essa camada é formada pelo produto de corrosão do aço que se transforma em uma camada aderente e protetora (Gubta e Birbilis, 2015). O aumento da resistência à corrosão para o aço inoxidável revestido é explicado pelo acúmulo do produto de corrosão do substrato nos poros, o que impede o contato da solução externa com o substrato, aumentando a resistência à corrosão (Tang et al., 2018). Nota-se que durante o período de 1000 h de imersão a resistência à corrosão do aço revestido teve algumas oscilações. Isso pode ser explicado pela remoção do produto de corrosão de alguns poros. Porém, com o tempo, esses poros são novamente preenchidos pelo produto de corrosão do substrato, regenerando a proteção do sistema substrato/revestimento.

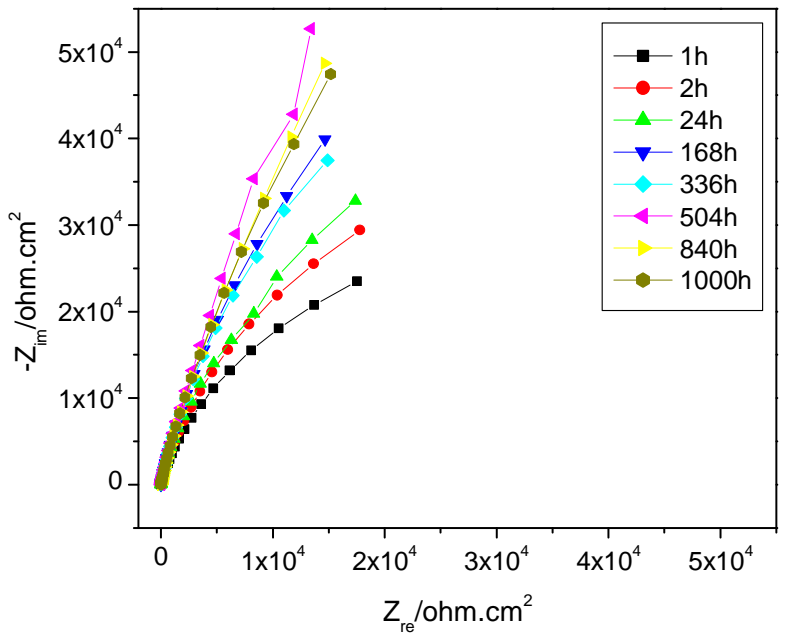

Figura 2 - Diagrama de Nyquist para o substrato puro $316 \mathrm{~L} \mathrm{em}$ meio de $\mathrm{NaCl} 3,0 \% \mathrm{~m} / \mathrm{v}$.

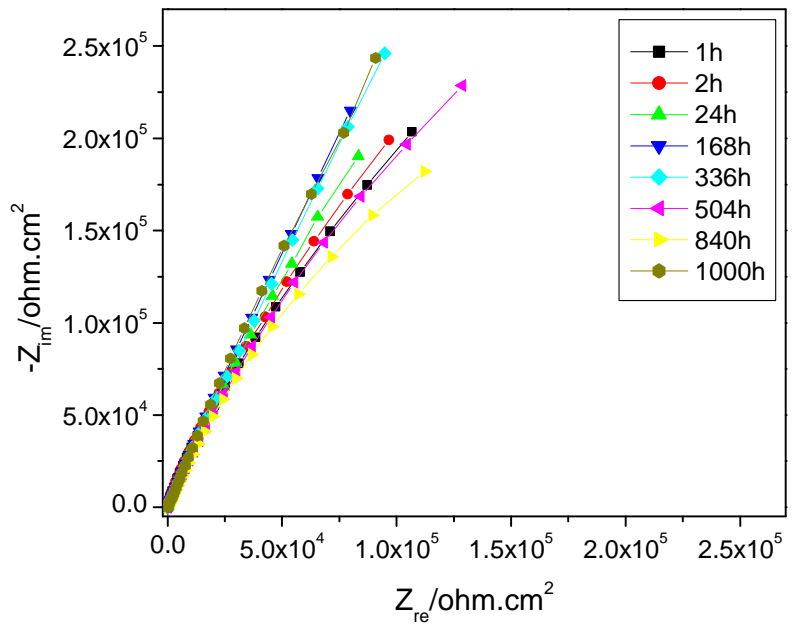

Figura 3 - Diagrama de Nyquist para o filme de $\mathrm{ZrN}$ depositado no aço 316L em meio de $\mathrm{NaCl} 3,0 \% \mathrm{~m} / \mathrm{v}$. 


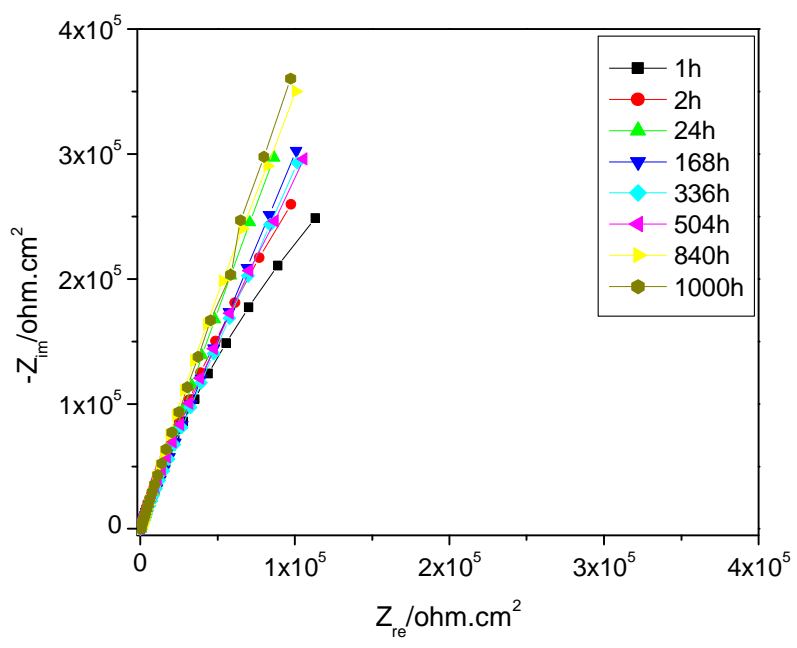

Figura 4 - Diagrama de Nyquist para o filme de $\mathrm{ZrSiN}$ depositado no aço $316 \mathrm{~L}$ em meio de $\mathrm{NaCl} 3,0 \% \mathrm{~m} / \mathrm{v}$.

Pelo diagrama de Bode, na figura 5, observa-se que, em baixa frequência $\left(10^{-2} \mathrm{~Hz}\right)$, o aço inoxidável $316 \mathrm{~L}$ sem revestimento possui menor ângulo de fase que as amostras revestidas. Segundo Lin e Duh (2009) para o diagrama de Bode em baixa frequência, quanto menor o ângulo de fase, mais resistivo o sistema, e por consequência, menos resistente à corrosão. E quanto maior o ângulo de fase, mais capacitivo o sistema e mais resistente à corrosão. Dessa forma, pode-se concluir que os filmes de $\mathrm{ZrN}$ e $\mathrm{ZrSiN}$ melhoram as propriedades de corrosão do substrato. Ainda, fazendo uma análise em baixa frequência, é possivel verificar que a adição de $\mathrm{Si}$ ao filme de $\mathrm{ZrN}$ melhora a resistência à corrosão do sistema substrato/filme, uma vez que o filme de $\mathrm{ZrSiN}$ possui maior ângulo de fase. Nota-se, também, que os sistemas com revestimento possuem um comportamento capacitivo com caráter resistivo residual. Já o aço sem revestimento possui um comportamento resistivo. Na figura 5, o módulo da impedância, que indica a resistência à troca de carga do sistema, mostra uma resistência na ordem de $10^{4}$ para o aço inoxidável $316 \mathrm{~L}$ sem vestimento e $10^{5}$ para o aço com revestimento. Também é possível verificar, pelo módulo de impedância, que o sistema com revestimento de $\mathrm{ZrSiN}$ possui uma resistência à corrosão maior que o de $\mathrm{ZrN}$.

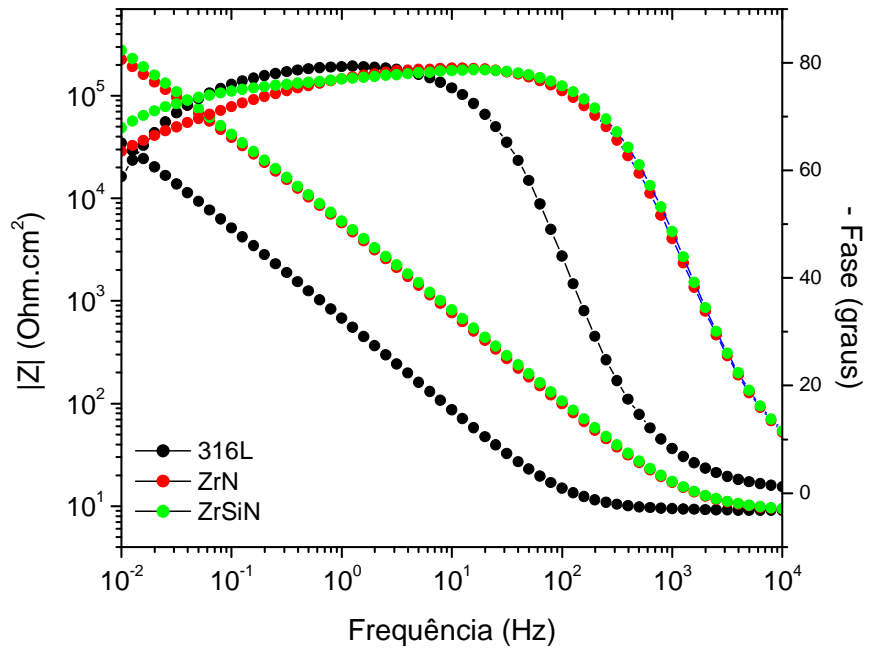

Figura 5 - Diagrama de Bode para as amostras 316L, $\mathrm{ZrN}$ e $\mathrm{ZrSiN}$ após $2 \mathrm{~h}$ de imersão.
$\mathrm{Na}$ tabela 2, é possível notar uma dureza de aproximadamente 16,0 GPa para os sistemas com $\mathrm{ZrN}$ e $\mathrm{ZrSiN}$. Dessa forma, conclui-se que a adição de Si na quantidade de 2,5 $\%$ at. não alterou a dureza do filme de $\mathrm{ZrN}$. Os valores encontrados estão de acordo com a literatura (MAE et al., 2001; FREITAS et al., 2015; SILVA et al., 2018). Os autores citados concordam que a dureza é afetada por vários fatores, como o nível de tensão de compressão, a orientação preferencial dos grãos nanocristalinos e o conteúdo da fase cristalina. Esses fatores são controlados pelos parâmetros de deposição dos filmes.

\section{Tabela 2 - Nanodureza dos filmes de $\mathrm{ZrN}$ e $\mathrm{ZrSiN}$ depositados no aço inoxidável 316L.

$\begin{array}{ccc}\text { Amostra } & \text { Dureza (GPa) } & \text { Desvio Padrão } \\ \mathrm{ZrN} & 16,5 & 1,4 \\ \mathrm{ZrSiN} & 16,4 & 0,3\end{array}$

$\mathrm{Na}$ figura 6 e 7 têm-se a análise morfológica dos revestimentos de $\mathrm{ZrN}$ e $\mathrm{ZrSiN}$ antes e após a imersão de $1000 \mathrm{~h}$, respectivamente. Na Figura 6 observa-se uma superfície homogênea, com marcas apenas das lixas que foram utlizadas na preparação da superfície do substrato. Uma vez que o revestimento é atômico, essas marcas ainda podem ser vistas após o revestimento ser depositado.

Na figura 7a, nota-se que após 1000 h de imersão parte do revestimento de $\mathrm{ZrN}$ se desprendeu do substrato. Já na figura $7 b$, não foi possível observar nenhuma alteração na superfície do revestimento de $\mathrm{ZrSiN}$, após imersão de 1000 h. Dessa forma, as imagens comprovam que a adição de silício ao filme de $\mathrm{ZrN}$, na porcentagem adicionada, possui efeito benéfico do ponto de vista de estabilidade química. Isso se deve, provavelmente, a diminuição da porosidade do filme.
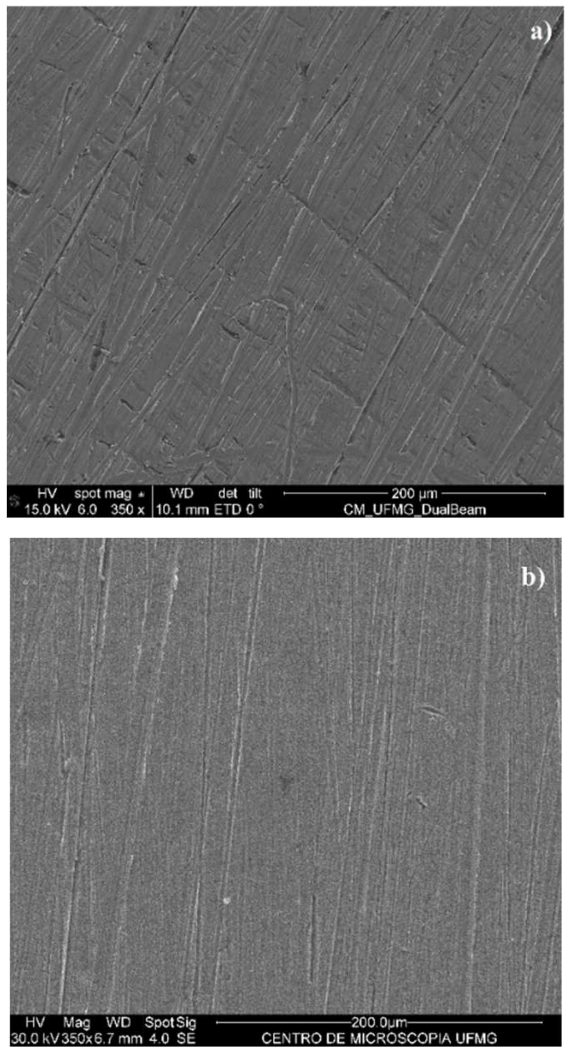

Figura 6 - Micrografia eletrônica de varredura antes do teste de imersão para as amostras com revestimento de a) $\mathrm{ZrN}$ e b) $\mathrm{ZrSiN}$. 

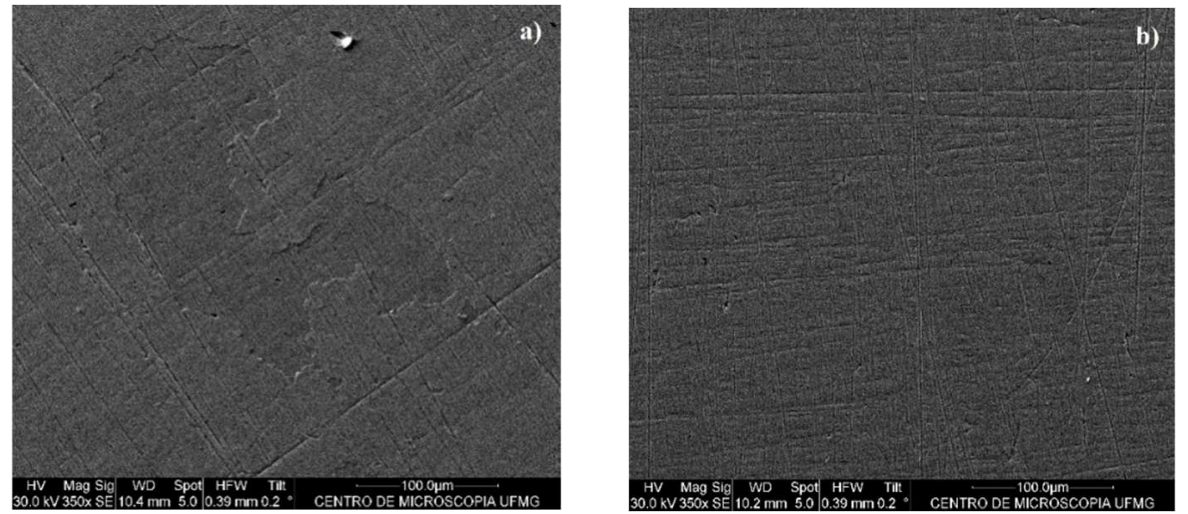

Figura 7 - Micrografia eletrônica de varredura após o teste de imersão de 1000 h para as amostras com revestimento de a) $\mathrm{ZrN}$ e b) $\mathrm{ZrSiN}$.

\section{CONCLUSÃO}

Os revestimentos de $\mathrm{ZrN}$ e $\mathrm{ZrSiN}$ melhoraram a resistência à corrosão eletroquímica do aço inoxidável 316L em meio a $\mathrm{NaCl}$ 3,0 \% m/v. Após 1000 h de imersão, pôdese verificar que a resistência à corrrosão do aço inoxidável $316 \mathrm{~L}$ com e sem revestimento aumenta com o tempo, devido a formação de uma camada protetiva de produto de corrosão. A adição de $\mathrm{Si}$ ao filme de $\mathrm{ZrN}$, na porcentagem adicionada, melhora as propriedades corrosivas do sistema, devido, provavelmente, a diminuição da porosidade do filme. Não se observou aumento da dureza com a adição de $2,5 \%$ de Si ao filme de $\mathrm{ZrN}$.

ALAT, E.; MOTTA, A.T.; COMSTOCK, R. J.; PARTEZANA, J.M.; WOLFE, D. E. Ceramic coating for corrosion (c3) resistance of nuclear fuel cladding. Surf. Coat. Technol, v. 281, p. 133-143, 2015.

CUBILLOS, G.I.; BETHENCOURT, M.; OLAYA, J.J.; ALFONSO, J.E.; MARCO, J.F. The influence of deposition temperature on microstructure and corrosion resistance of $\mathrm{ZrO}_{\mathrm{x}} \mathrm{N}_{\mathrm{y}} / \mathrm{ZrO}_{2}$ coatings deposited using $\mathrm{RF}$ sputtering. Applied Surface Science, v. 309, p. 181-187, 2014.

CUI, X.; JIN, G.; HAO, J.; LI, J.; GUO, T. The influences of Si content on biocompatibility and corrosion resistance of $\mathrm{Zr}-\mathrm{Si}-\mathrm{N}$ films. Surf. Coat. Technol., v. 228, p. S524-S528, 2013.

FREITAS, F.G.R.; HÜBLERB, R.; SOARESC, G.; CONCEIÇÃO, A.G.S.; VITÓRIA, E.R.; CARVALHO, R.G.; TENTARDINI, E.K. Structural and mechanical properties of $\mathrm{Zr}$ $\mathrm{Si}-\mathrm{N}$ thin films prepared by reactive magnetron sputtering. Mater. Res., v. 18 (supply2), p. 30-34, 2015.

\section{AGRADECIMENTOS}

À CAPES e ao CNPq pelo auxílio financeiro.

\section{R E F E R E N C I A S}

GUPTA, R. K.; BIRBILIS, N. The influence of nanocrystalline structure and processing route on corrosion of stainless steel: A review. Corros. Sci., v.92, p. 1-15, 2015.

HARRISON, R.W.; W.E. LEE. Processing and properties of $\mathrm{ZrC}, \mathrm{ZrN}$ and $\mathrm{ZrCN}$ ceramics: a review. Adv. Appl. Ceram., v. 115, p. 294307, 2016.

LIN, C. H.; DUH, J. G. Electrochemical impedance spectroscopy (EIS) study on corrosion performance of CrAlSiN coated steels in 3.5 wt.\% NaCl solution. Surf. Coat. Technol., v. 204, p. 784-787, 2009.

MAE, T.; NOSE, M.; ZHOU, M.; NAGAE, T.; SHIMAMURA, K. The effects of Si addition on the structure and mechanical properties of $\mathrm{ZrN}$ thin films deposited by an r.f. reactive sputtering method. Surf. Coat. Technol., v.142-144, p. 954-958, 2011.

MILANI, R. Nitretação a plasma de zircônia parcialmente estabilizada. Dissertação de mestrado. Universidade de Caxias do Sul, Caxias do Sul, 2009.

SAFI, I. Recent aspects concerning DC reactive magnetron sputtering of thin films: a review. 
Surf. Coat. Technol., v. 127, p. 203-219, 2000.

SILVA NETO, P.C.; FREITAS, F.G.R.; FERNANDEZ, D.A.R.; CARVALHO, R.G.; FELIX, L.C.; TERTO, A.R.; HUBLER, R.; MENDES, F.M.T.; SILVA JUNIOR, A.H.; TENTARDINI, E.K. Investigation of microstructure and properties of magnetron sputtered $\mathrm{Zr}-\mathrm{Si}-\mathrm{N}$ thin films with different $\mathrm{Si}$ content. Surf. Coat. Technol, v.353, p.355363, 2018.

TANG, Q-L. Mechanical property evaluation of $\mathrm{ZrSiN}$ films deposited by a hybrid superimposed high power impulse- medium frequency sputtering and RF sputtering system, Surf. Coat. Technol (2018), https://doi.org/10.1016/j.surfcoat.2018.03.1 03.

YALAMANCHILI, K.; JIMÉNEZ-PIQUÉ, E.; PELCASTRE, L.; BAKOGLIDIS, K.D.; ROA, J.J.; JOHANSSON JÖESAAR, M.P.; PRAKASH, B.; GHAFOOR, N.; ODÉN, M. Influence of microstructure and mechanical properties on the tribological behavior of reactive arc deposited $\mathrm{Zr}-\mathrm{Si}-\mathrm{N}$ coatings at room and high temperature. Surf. Coat. Technol, v.304, p. 393-400, 2016. 